\title{
TNF-TNFR2 Signaling Inhibits Th2 and Th17 Polarization and Alleviates Allergic Airway Inflammation
}

\author{
Juan Peng Xiao-Ming Li Guo-Rui Zhang YiCheng Xi Chen Wen Gu \\ Xue-Jun Guo \\ Department of Respiratory Medicine, Xinhua Hospital, School of Medicine, Shanghai Jiao Tong University, \\ Shanghai, China
}

\section{Keywords}

Allergic airway inflammation - TNF-TNFR2 signaling ·

CD4+ T lymphocyte

\begin{abstract}
Background: TNF-TNFR2 signaling has been indicated to be involved in CD4+ T lymphocyte differentiation. However, its role in allergic airway inflammation is not well understood. Objectives: The aim of this study was to investigate the role of TNF-TNFR2 signaling in allergic airway inflammation. Methods and Results: In this study, we used an allergen-induced asthma model to show that TNF-TNFR2 signaling alleviated allergic airway inflammation by reducing the airway infiltration of eosinophils and neutrophils. Activated TNFTNFR2 signaling decreased the expression of Th2 and Th17 cytokines in serum and bronchoalveolar lavage fluid. Furthermore, TNF-TNFR2 signaling inhibited Th2 and Th17 polarization but promoted Th1 and CD4+CD25+ T cell differentiation in vivo. Conclusions: Our study indicates that TNFTNFR2 signaling alleviates allergic airway inflammation through inhibition of Th2 and Th17 cell differentiation.
\end{abstract}

๑) 2019 S. Karger AG, Basel

J.P. and X.-M.L. contributed equally to this work.

Edited by: H.-U. Simon, Bern.
(C) 2019 S. Karger AG, Basel

KARGER

E-Mail karger@karger.com

www.karger.com/iaa

\section{Introduction}

Allergic asthma is a common chronic airway disease that affects millions of people worldwide $[1,2]$. A vast array of cells, such as eosinophils, neutrophils, mast cells, and $\mathrm{T}$ lymphocytes, are involved in the airway inflammation of asthma [3-5]. Th2 cells, as well as their cytokines IL-4, IL-5, and IL-13, have been proven to underlie the process of allergic asthma [6,7]. Recent studies have shown that the IL-17 secreted by Th17 cells is able to induce neutrophil recruitment $[8,9]$. Moreover, overexpression of IL-17 has been found in sputum, bronchoalveolar lavage fluid (BALF), and lung tissue in chronic allergic airway inflammation [10-12]. Our previous studies showed that Th17, similar to Th2, is also associated with airway inflammation in asthma $[13,14]$. Therefore, the effective inhibition of Th2 and Th17 cell differentiation may be important for controlling airway inflammation in asthma.

TNFR2 (tumor necrosis factor receptor 2) is a member of the tumor necrosis factor super family. Compared to TNFR1, TNFR2 is expressed in a more limited manner on certain populations of lymphocytes, and it is involved in the differentiation and survival of lymphocytes [15]. As Chen and Oppenheim $[16,17]$ reported, TNFR2 promotes the activation, expansion, and survival of Treg

Dr. Xue-Jun Guo

Department of Respiratory Medicine, Xinhua Hospital

School of Medicine, Shanghai Jiao Tong University

1665 Kongjiang Road, Shanghai 200092 (China)

E-Mail guoxuejun@xinhuamed.com.cn 


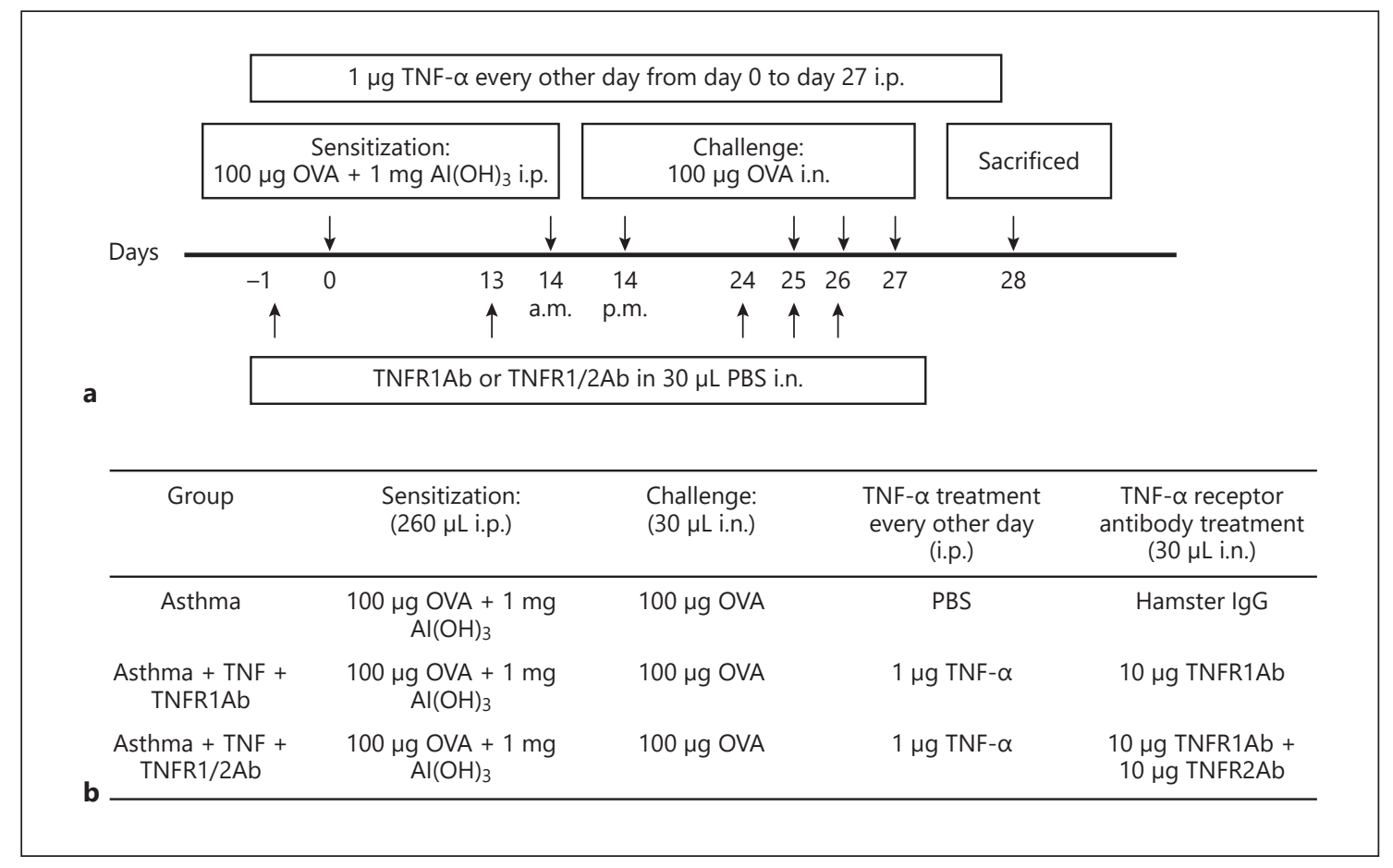

Fig. 1. Timetable for OVA-induced allergic airway inflammation mouse model and TNFR2 signaling intervention. a Mice were sensitized intraperitoneally (i.p.) with $100 \mu \mathrm{g}$ of OVA and $1 \mathrm{mg}$ of $\mathrm{Al}(\mathrm{OH})_{3}$ on days 0 and 14 and challenged intranasally (i.n.) with $100 \mu \mathrm{g}$ of OVA on days 25,26 , and 27 . For TNFR2 signaling activation, the mice were treated with $1 \mu \mathrm{g}$ of recombinant murine TNF- $\alpha$ intraperitoneally every other day. In addition, the mice were treated with $10 \mu \mathrm{g}$ of TNFR1 blocking antibody intranasally
1 day before each sensitization and challenge. For TNFR2 signaling inhibition, the mice were treated with $10 \mu \mathrm{g}$ of TNFR1 blocking antibody and $10 \mu \mathrm{g}$ of TNFR2 blocking antibody intranasally 1 day before each sensitization and challenge. b For TNF- $\alpha$ treatment control, $30 \mu \mathrm{L}$ of PBS was used as a control. For TNF- $\alpha$ receptor antibody treatment control, $10 \mu \mathrm{g}$ of hamster IgG was used as a control. cells, especially in the inflammatory environment. Furthermore, Candel et al. [18] demonstrated that TNFR2 deficiency may exacerbate oxidative stress-induced inflammation. Another study indicated that lack of TNFR2aggravated radiation induced inflammation of the nervous system [19]. Our recent study also demonstrated that impaired TNFR2 signaling promotes Th2 and Th17 polarization and exacerbates allergic airway inflammation [20]. However, whether activated TNFR2 signaling can alleviate allergic airway inflammation is unclear.

The current findings demonstrated that activated TNFR2 signaling alleviated airway inflammation by decreasing eosinophil and neutrophil recruitment. Moreover, TNFR2 signaling activation reduced Th2 and Th17 cytokine expression. Furthermore, TNF-TNFR2 signaling inhibited Th2 and Th17 polarization but promoted Th1 and CD4+CD25+ T cell differentiation in vivo. Our results thus indicate a critical role of TNFR2 signaling in allergic airway inflammation through regulation of Th2 and Th17 differentiation.

\section{Materials and Methods}

Mice

Six-week-old female BALB/c mice were purchased from Shanghai SLAC Co. (Shanghai, China). All animal care and handling protocols were approved by the Ethics Committee of Xinhua Hospital Affiliated to Shanghai Jiao Tong University School of Medicine. All of the experiments were carried out in accordance with the Guide for the Care and Use of Laboratory Animals.

\section{Asthma Model and TNFR2 Signaling Intervention}

We generated an asthma model according to previously published protocols [13]. Briefly, mice were sensitized intraperitoneally twice on days 0 and 14 with $100 \mu \mathrm{g}$ of OVA (Sigma-Aldrich, St. Louis, MO, USA) and $1 \mathrm{mg}$ of $\mathrm{Al}(\mathrm{OH})_{3}$ (Thermo Scientific, Waltham, MA, USA). On days 25, 26, and 27, the mice were challenged with $100 \mu \mathrm{g}$ of OVA intranasally. For TNFR2 signaling ac- 
tivation, the mice were treated with $1 \mu \mathrm{g}$ of recombinant murine TNF- $a$ (PeproTech, Rocky Hill, NJ, USA) intraperitoneally every other day. In addition, the mice were treated with $10 \mu \mathrm{g}$ of TNFR1 blocking antibody (TNFR type I/p55, clone No. 55R-593; Biolegend, San Diego, CA, USA) intranasally 1 day before each sensitization and challenge. For TNFR2 signaling inhibition, the mice were treated with $10 \mu \mathrm{g}$ of TNFR1 blocking antibody and $10 \mu \mathrm{g}$ of TNFR2 blocking antibody (TNFR type II/p75, clone No. TR7554.7; Biolegend) intranasally 1 day before each sensitization and challenge, as shown in Figure 1.

\section{Collection of Serum and BALF}

Mice were sacrificed by an overdose of $100 \mathrm{mg} / \mathrm{kg}$ of pentobarbital intraperitoneally $24 \mathrm{~h}$ after the final challenge. Blood was collected by cardiac puncture. After centrifugation at 3,000 rpm for $15 \mathrm{~min}$ at $4{ }^{\circ} \mathrm{C}$, serum was collected and stored at $-80^{\circ} \mathrm{C}$ for cytokine analysis. We used $0.8 \mathrm{~mL}$ of cold phosphate-buffered saline for BALF, which was collected and centrifuged at $400 \mathrm{~g}$ for $5 \mathrm{~min}$ at $4{ }^{\circ} \mathrm{C}$. The supernatant was collected and stored at $-80^{\circ} \mathrm{C}$, while the precipitate was analyzed for cellular composition using WrightGiemsa staining.

\section{Lung Histology}

The right lungs were removed and fixed in $4 \%$ buffered paraformaldehyde for at least $24 \mathrm{~h}$ at room temperature. The paraffin lung sections were stained with hematoxylin and eosin (HE) or periodic acid-Schiff (PAS) and analyzed by microscope under $\times 200$ magnification.

\section{Cytokine Analysis}

Lymphocytes derived from the spleen were cultured in vitro and stimulated with OVA $(10 \mu \mathrm{g} / \mathrm{mL})$ for 3 days. Then, the cultured supernatant was collected and stored at $-80^{\circ} \mathrm{C}$ for cytokine analysis. IL-4, IL-5, IL-10, IFN- $\gamma$, and IL-17A from serum, BALF, and cultured supernatant were analyzed by enzyme-linked immunosorbent assay kits (eBioscience, San Diego, CA, USA) according to the manufacturer's instructions. These results were analyzed by a Bio-Rad Bio-Plex instrument (Bio-Rad, Hercules, CA, USA).

\section{Flow Cytometry}

For CD4+ T cell polarization analysis, lymphocytes from spleen and lung-draining mediastinal lymph nodes were separated and stimulated with a leukocyte activation cocktail (BD Bioscience, Franklin Lakes, NJ, USA) at $2 \mu \mathrm{L} / \mathrm{mL}$ for $5 \mathrm{~h}$. CD4-FITC and CD25-PE (eBioscience) were used to label CD4+CD25+ T cells. For intracellular cytokine staining, lymphocytes were permeabilized and fixed with a Cytofix/Cytopermea Kit (eBioscience). IFN$\gamma$-APC, IL-4-PE, and IL-17-PerCP-cy5.5 (eBioscience) were used to label Th1, Th2, and Th17 cells, respectively. All the samples were analyzed by BD FACSCalibur (BD Bioscience).

\section{Real-Time PCR}

Total RNA of lymphocytes from spleens was extracted using TRIzol reagent (Takara Biotechnology, Dalian, China), and cDNA was synthesized using a PrimeScript ${ }^{\mathrm{TM}} \mathrm{RT}$ reagent kit (Takara Biotechnology, Dalian, China). Ask1 and Bmx, which are the downstream genes of TNFR1 and TNFR2, respectively, were examined with an ABI 7500 real-time PCR system using a SYBR Premix Ex Taq TM II kit. The following primer pairs were used: Ask1 forward: 5'-CAAATCAGACAGTCCGACGG-3'; reverse: 5'-GTG-

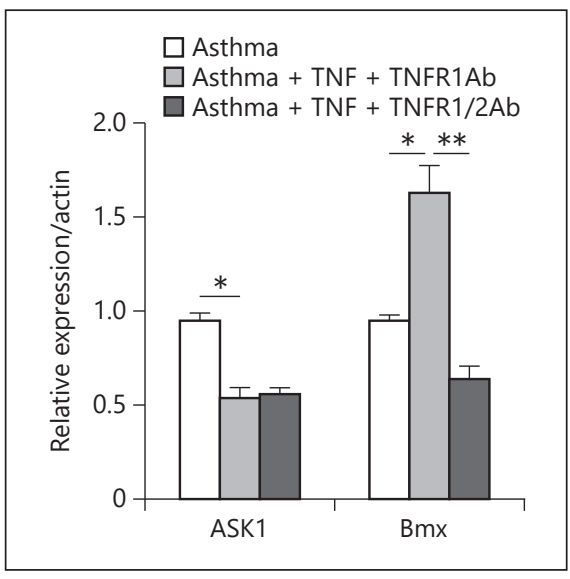

Fig. 2. TNF- $\alpha$ and TNFR1 blocking antibody administration enhanced the gene expression of Bmx. The expression of the TNFR2specific downstream gene Bmx was increased in the TNF- $\alpha$ and TNFR1 blocking antibody treatment group compared with the control group. The expression of the TNFR1-specific downstream gene Ask1 was decreased in the TNF- $\alpha$ and TNFR1 blocking antibody treatment group compared with the control groups. Data are expressed as the mean \pm SEM from 6 mice in each group. ${ }^{*} p<0.05$, $* * p<0.01$.

CATTCTGGGAGTCATGG-3' ${ }^{\prime}$. Bmx forward: $5^{\prime}$-GGAAAGTTCCTGTGTTGCCA-3' ; reverse: $5^{\prime}$-TGGGAGCTCTGTGTTTC TCA-3'. Actin forward: $5^{\prime}$-GTGCTATGTTGCTCTAGACTTCG-3';

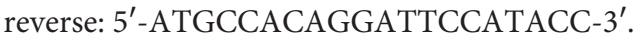

\section{Western Blotting}

Lymphocytes from spleens were lysed by means of radio-immunoprecipitation assay buffer containing protease and phosphatase inhibitor cocktails (Sigma-Aldrich). Protein samples $(50 \mu \mathrm{g})$ were run on sodium dodecyl sulfate-polyacrylamide gels and transferred to polyvinylidene difluoride membranes. After incubation with a blocking buffer (5\% nonfat milk in $10 \mathrm{~mm}$ Tris- $\mathrm{HCl}$, $\mathrm{pH} 7.5,100 \mathrm{mM} \mathrm{NaCl}, 0.1 \%$ [w/v] Tween-20) for $2 \mathrm{~h}$, the membrane was incubated with primary antibodies for T-bet, GATA3, FoxP3, ROR $\gamma$ (all 1:300; Boster, Wuhan, China), or actin (1:2,000; Beyotime, Shanghai, China) and then visualized with a horseradish peroxidase-conjugated secondary antibody (1:2,000; Beyotime) and enhanced chemiluminescence agents. The relative quantities of proteins were estimated by scanning densitometry (ChemiDoc XRS Systems, Bio-Rad Laboratories Inc.) and analyzed by Image Lab 5.0 software (Bio-Rad Laboratories Inc.).

\section{Statistical Analysis}

Statistical analysis was performed using SPSS 17.0 software. Data are expressed as the mean \pm SEM. An independent $t$ test was used for the comparisons between two groups. For multiple comparisons one-way ANOVA was used first and, if a significant difference was detected, an LSD post hoc test was performed between pairs of groups. A $p$ value $<0.05$ was considered statistically significant.
The Role of TNF-TNFR2 Signaling in

Allergic Airway Inflammation
Int Arch Allergy Immunol 2019;178:281-290 DOI: $10.1159 / 000493583$ 


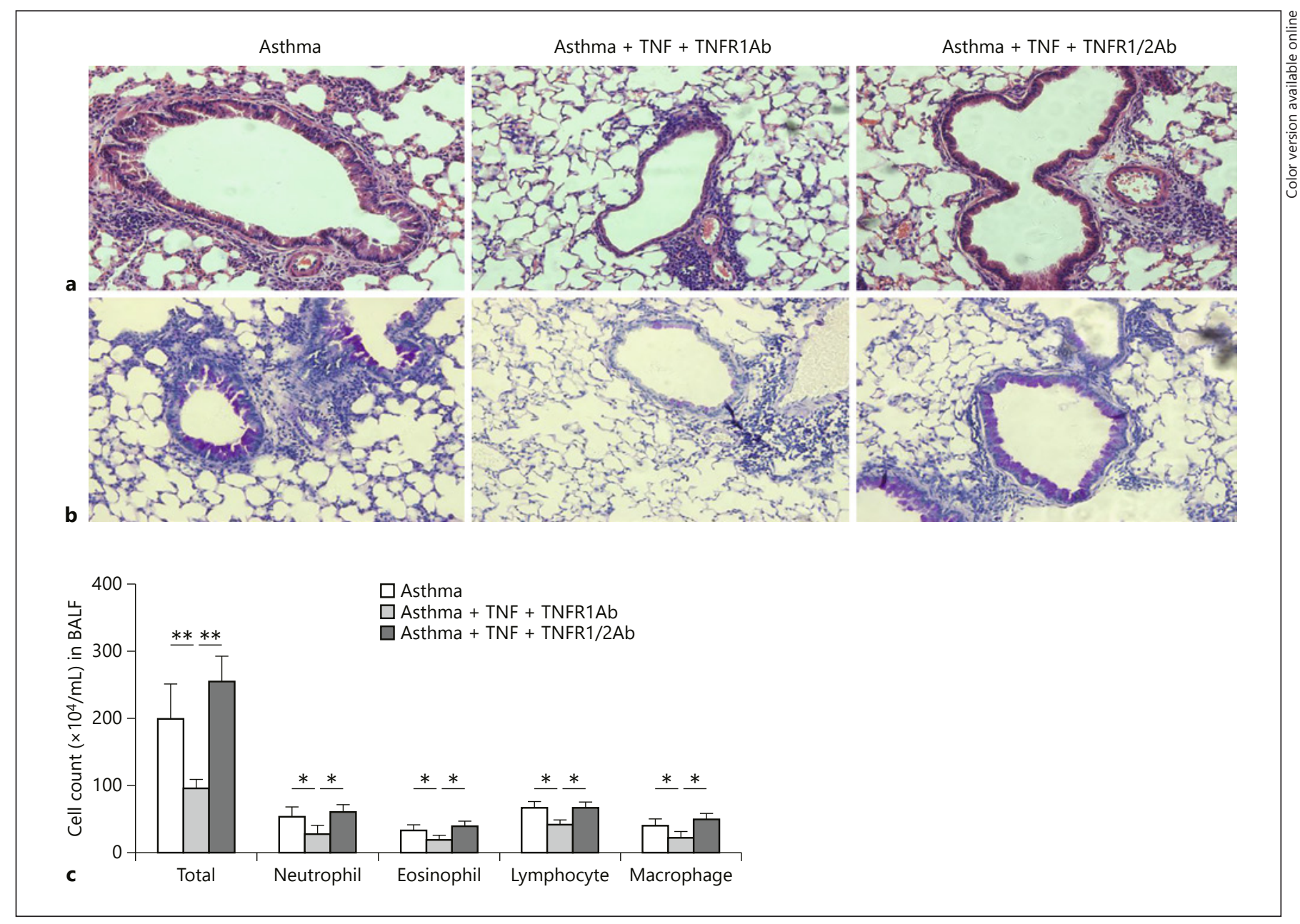

Fig. 3. TNF-TNFR2 signaling activation alleviated airway inflammation. a Lung histology was analyzed by HE staining. b Lung histology was analyzed by PAS staining. $\mathbf{c}$ The total cell numbers and cell composition in BALF were assessed by cytospin with Wright-Giemsa staining. Data are expressed as the mean \pm SEM ( $n=6$ mice per group). ${ }^{*} p<0.05,{ }^{* *} p<0.01$.

\section{Results}

TNFR2 Signaling Is Activated Effectively by TNF- $\alpha$ and TNFR1 Blocking Antibody

To identify whether TNFR2 signaling was activated by TNF- $\alpha$ and TNFR1 blocking antibody, we first examined the Ask1 and Bmx gene expression. As shown in Figure 2 , the expression of the Bmx gene was dramatically increased in the spleen lymphocytes from TNF- $\alpha$ and TNFR1 blocking antibody-treated mice compared with the expression in untreated mice. Additionally, the expression of the Ask1 gene was significantly decreased in the spleen lymphocytes from TNF- $\alpha$ and TNFR1 blocking antibody-treated mice compared with that from un- treated mice. These results indicate that TNFR2 signaling was activated effectively by TNF- $\alpha$ and TNFR1 blocking antibody. Furthermore, the expression of both Ask1 and Bmx genes were decreased when TNFR1 and TNFR2 signaling were inhibited simultaneously.

\section{TNF-TNFR2 Signaling Activation Alleviates Airway Inflammation}

Our previous study showed that impaired TNFR2 signaling aggravated airway inflammation [20]. To evaluate the effect of TNF-TNFR2 signaling activation on airway inflammation, we first activated TNF-TNFR2 signaling by TNF- $\alpha$ and TNFR1 blocking antibody. As shown in Figure $3 \mathrm{a}$ and $\mathrm{b}$, the inflammatory cell infiltration and 


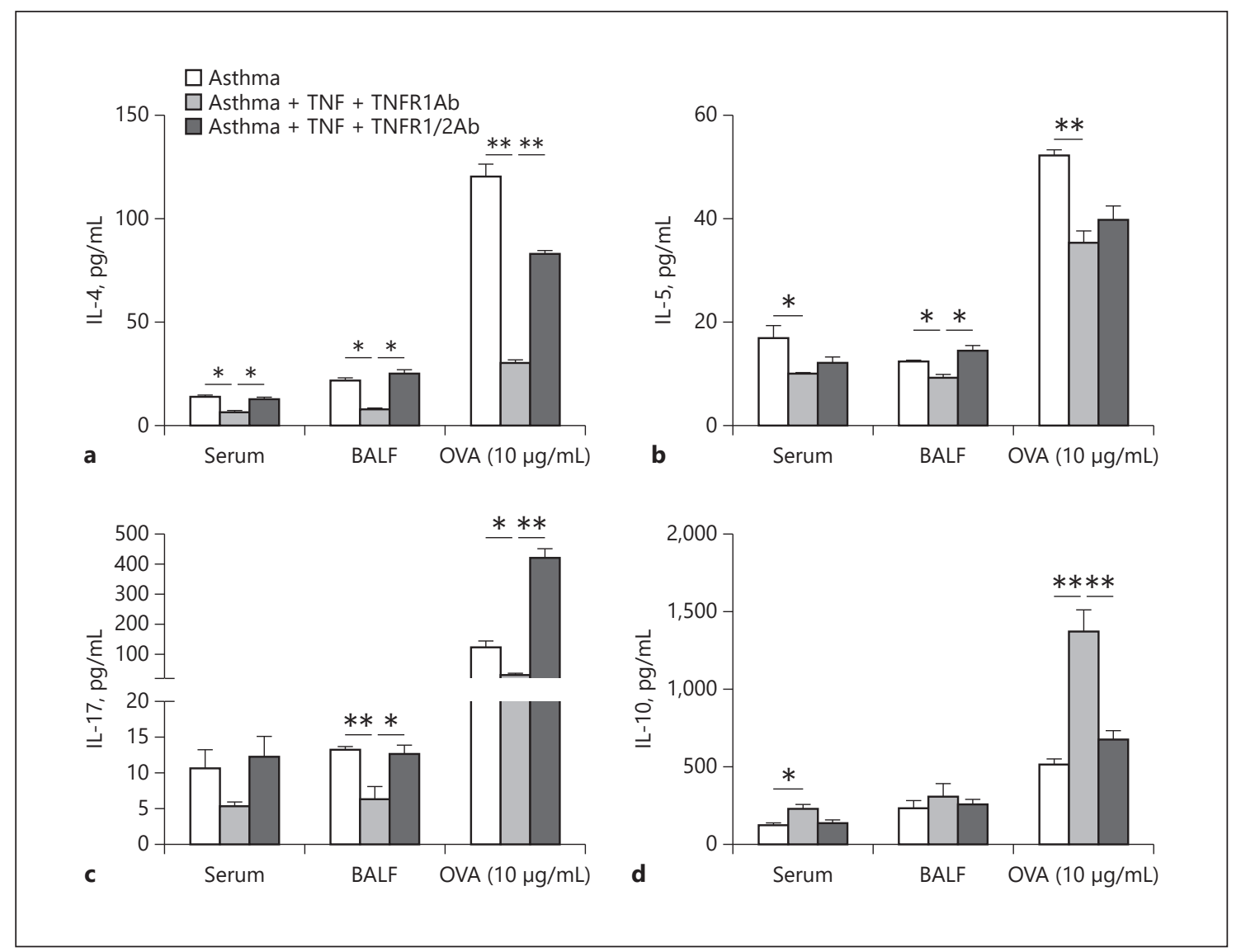

Fig. 4. TNF-TNFR2 signaling activation affected the expression of cytokines. The expression of IL4, IL-5, IL-17, and IL-10 in serum, BALF, and cell culture supernatant was analyzed by ELISA. Data are expressed as the mean $\pm \operatorname{SEM}\left(n=6\right.$ mice per group). ${ }^{*} p<0.05,{ }^{* *} p<0.01$.

mucus secretion were greatly inhibited in the lungs from the TNF-TNFR2 activation group compared with the control group. Furthermore, inflammatory cells in the BALF were typed and quantified by Wright-Giemsa staining. As shown in Figure 3c, the eosinophils and neutrophils were both significantly decreased in the TNFTNFR2 activation group compared with the untreated group. Next, when TNFR1 and TNFR2 signaling were inhibited simultaneously, as shown in Figure $3 a-c$, the inhibited airway inflammation was restored. Taken together, these findings suggest that TNF-TNFR2 signaling activation alleviates airway inflammation.

\section{TNF-TNFR2 Signaling Activation Inhibits Th2 and Th17 Cytokine Expression}

Since Th2 and Th17 cytokines regulate airway inflammation by the recruitment of eosinophils and neutrophils, respectively, we subsequently investigated whether
TNF-TNFR2 signaling activation has an impact on type 2 and type 17 cytokine expression. As shown in Figure $4 \mathrm{a}-\mathrm{d}$, the expression of IL- 4 and IL-5 were significantly reduced in the serum and BALF, as well as in cell culture supernatant from the TNF-TNFR2 signaling activation group compared with the control group. Moreover, the production of IL-17, a Th17 cytokine, was also significantly decreased in the BALF and cell culture supernatant compared with the untreated group. However, IL-10, a typical tolerogenic cytokine [21], which can inhibit proinflammatory cytokine synthesis, was significantly increased compared with the untreated group. Similarly, when TNFR1 and TNFR2 signaling were inhibited simultaneously, the expression of proinflammatory cytokines IL-4, IL-5, and IL-17 were increased, while the expression of IL-10 was decreased. These results suggest that TNFTNFR2 signaling activation inhibits proinflammatory cytokine expression. 


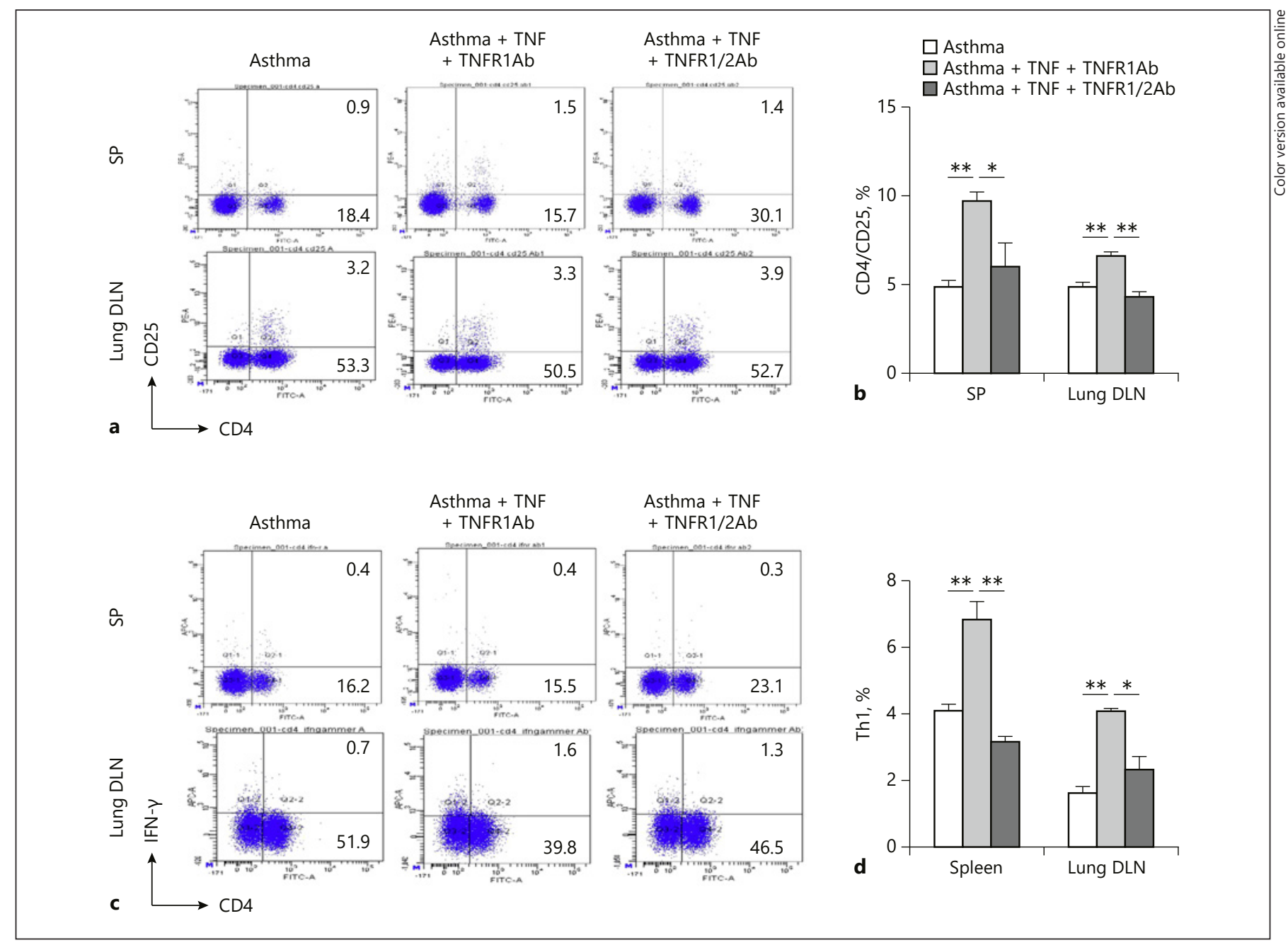

Fig. 5. TNF-TNFR2 signaling activation influenced CD4+ T lymphocyte differentiation. Lymphocytes from spleen and lung-draining lymph nodes were restimulated with a leukocyte activation cocktail. Treg (a, b), Th1 (c, d), Th2 (e,f), and Th17 (g, h) cell differentiation was analyzed by flow cytometry. The data shown in the upper right quadrants represent the percentages of positive cells. The percentages of each subset of total CD4+ T cells were calculated. Data are expressed as the mean \pm SEM ( $n=6$ mice per group). ${ }^{*} p<0.05,{ }^{* *} p<0.01$.

(Figure continued on next page.)
TNF-TNFR2 Signaling Activation Influences CD4+ T Lymphocyte Differentiation in vivo

Chen and Oppenheim [16] reported that TNFR2 is essential for the stabilization of CD4+FoxP3+ Tregs in vitro. Our previous study demonstrated that impaired TNF-TNFR2 signaling may also promote Th2 and Th17 polarization [20]. Therefore, we next examined the impact of TNF-TNFR2 signaling activation on CD4+ T lymphocyte differentiation in vivo. As shown in Figure $5 \mathrm{a}-\mathrm{d}$, the differentiation of Th1 and CD4+CD25+ T cells from spleen and lung-draining lymph nodes were significantly enhanced in the TNF-TNFR2 signaling activation group compared with the control group. In con- trast, Th2 and Th17 cell differentiation were significantly inhibited by TNF-TNFR2 signaling activation (Fig. 5e-h). Next, when TNFR1 and TNFR2 signaling were inhibited simultaneously, differentiation of Th1 and CD4+CD25+ T cells was inhibited, while differentiation of Th2 and Th17 cells was enhanced. Thus, TNFTNFR2 signaling can influence CD4+ T lymphocyte differentiation.

\section{TNF-TNFR2 Signaling Activation Affects}

Transcription Factor Expression

To understand the potential mechanism of the effect of TNF-TNFR2 signaling activation on CD4+ T lym- 


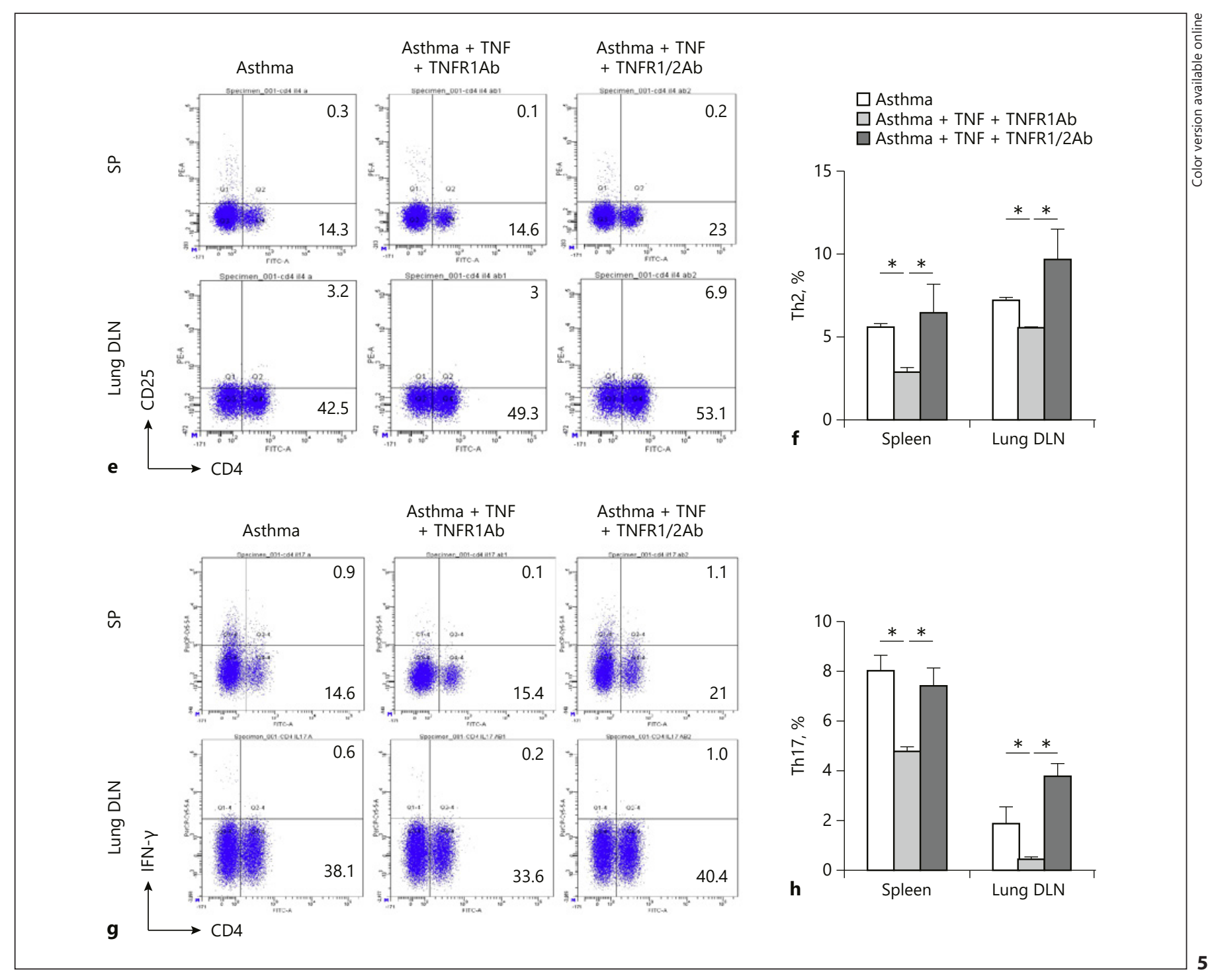

phocyte differentiation, we next examined the expression of transcription factors, such as T-bet, GATA3, FoxP3, and ROR $\gamma$. As shown in Figure 6, TNF-TNFR2 signaling activation promoted FoxP3 and T-bet expression but reduced GATA3 and ROR $\gamma$ expression. Consistently, when TNFR1 and TNFR2 signaling were inhibited simultaneously, we observed opposite results. Taken together, the results indicate that TNF-TNFR2 signaling activation can inhibit differentiation of Th2 and Th17 cells by decreasing GATA3 and ROR $\gamma$ expression.

\section{Discussion}

TNF- $\alpha$ is a cytokine with various immune regulatory functions [22]. The role of TNF- $\alpha$ in inflammatory disease remains controversial. Previous studies have shown that TNF- $\alpha$ is a proinflammatory mediator and plays an important role in the process of inflammation [23, 24]. However, other studies have also proved that TNF- $\alpha$ has an anti-inflammatory effect $[25,26]$. It has been shown that TNF- $\alpha$ functions by TNFR1 and TNFR2 signaling, respectively. TNFR1 signaling plays a major role in the cytotoxic, proinflammatory, and apoptotic effects, while TNFR2 signaling predominantly mediates lymphocyte activation and proliferation [22]. Recently, TNF-TNFR2 


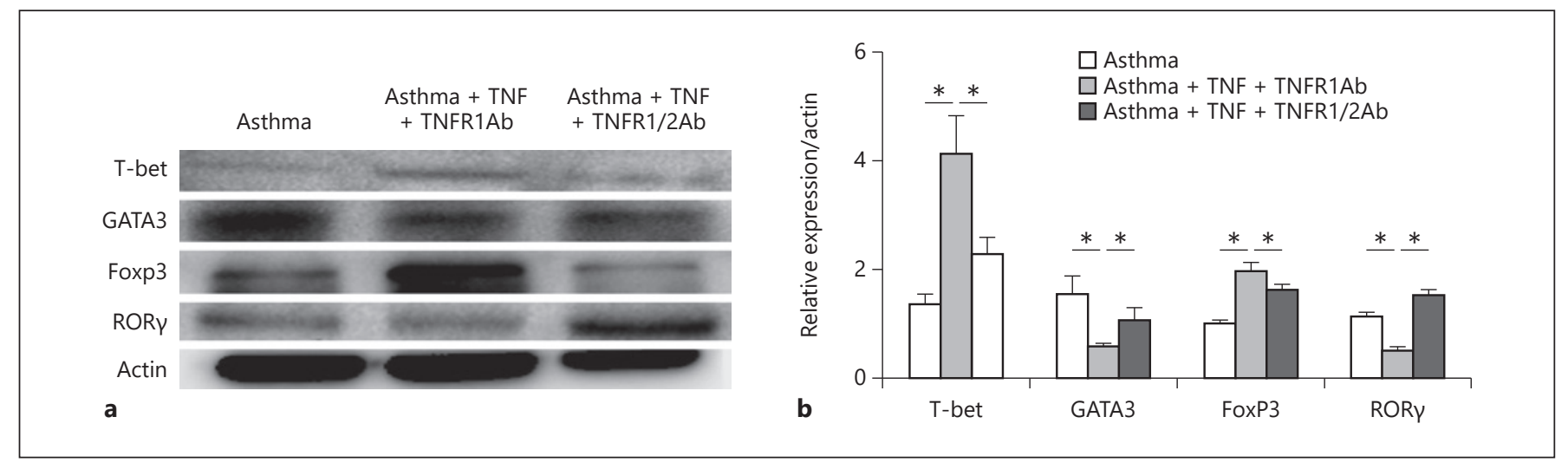

Fig. 6. TNF-TNFR2 signaling affected the expression of transcription factors during the process of CD4+ T lymphocyte differentiation. The protein expression of each transcription factor in lymphocytes derived from the spleen of each group was analyzed by
Western blotting. a Representative Western blots. b Densitometry analysis of T-bet, GATA3, FoxP3, and ROR $\gamma$ normalized to actin protein from 3 independent experiments. Data are expressed as the mean $\pm \operatorname{SEM}$ ( $n=6$ mice per group). ${ }^{*} p<0.05$. signaling has been proven to be involved in the differentiation of CD4+ T lymphocytes $[15,16]$. As reported by $\mathrm{Wu}$ et al. [27], the administration of TNF- $\alpha$ to young adult nonobese diabetic mice every other day for 3 weeks can reduce the incidence of diabetes, which may be attributed to the increased number of CD $4+C D 25+\mathrm{T}$ cells in the thymus and spleen. Chen et al. [28] reported that TNF- $\alpha$ administration expands CD4+CD25+ T cells via TNFR2 signaling in vitro. Our previous study has also demonstrated that impaired TNF-TNFR2 signaling exacerbates airway inflammation by promoting Th2 and Th17 polarization. Therefore, we hypothesized that the activated TNF-TNFR2 signaling may alleviate allergic airway inflammation. In the current study, TNFR2 signaling was activated by administrating TNF- $\alpha$ and a TNFR1 blocking antibody simultaneously. Our results demonstrate that TNFR2 signaling activation alleviated airway inflammation and inflammatory cytokine expression by inhibition of Th2 and Th17 cell differentiation.

Asthma is characterized by high mucus secretion and infiltration of airway inflammatory cells, such as eosinophils, neutrophils, lymphocytes, and macrophages. In this study, TNFR2 signaling activation profoundly reduced the mucus production and inflammatory cell infiltration in the airway. These results are consistent with the finding of Maier et al. [29], showing that the activation of TNFR2 signaling protects oligodendrocyte progenitor cells against oxidative stress. Moreover, oligodendroglial TNFR2 mediates membrane TNF-dependent repair in experimental autoimmune encephalomyelitis [30].
Therefore, these findings suggest that TNFR2 signaling activation alleviates airway inflammation in asthma.

Eosinophilia and neutrophilia are associated with type 2 and type 17 immune responses, which are characterized by the production of Th2 and Th17 cytokines, respectively. As expected, TNFR2 signaling activation dramatically reduced the production of the proinflammatory cytokines IL-4, IL-5, and IL-17, but enhanced the production of the anti-inflammatory cytokine IL-10 in serum, BALF, and splenocyte cultures. These results collectively suggest that the alleviation of airway inflammation by TNFR2 signaling activation may be attributed to an altered cytokine expression.

CD4+ T lymphocyte differentiation plays an important role in airway inflammation. Recently, TNFR2 signaling has been confirmed to be involved in CD4+ T lymphocyte differentiation. Here, we found that TNFR2 signaling activation inhibited Th2 and Th17 polarization but promoted Th1 and CD4+CD25+ T cell differentiation. Furthermore, we examined the expression levels of transcription factors of each CD4+ T cell subset to clarify the potential mechanism underlying TNFR2 signaling activation in the regulation of CD4+ T lymphocyte differentiation. We found that TNFR2 signaling activation inhibited GATA3 and ROR $\gamma$ expression but promoted FoxP3 and T-bet expression. Together, these data suggest that TNFR2 signaling regulates CD4+ T cell differentiation through activation of different transcription factors.

In conclusion, TNF-TNFR2 signaling activation alleviated airway inflammation, and a potential mechanism may involve the inhibited polarization of Th2 and Th17. 


\section{Acknowledgement}

We thank the staff at the Center for Medical Research of Xinhua Hospital for their assistance.

\section{Statement of Ethics}

All animal care and handling protocols were approved by Ethics Committee of Xinhua Hospital Affiliated to Shanghai Jiao Tong University School of Medicine. All of the experiments were carried out in accordance with the Guide for the Care and Use of Laboratory Animals.

\section{Disclosure Statement}

The authors have no conflicts of interest to declare.

\section{Funding Sources}

This work was supported by the National Natural Sciences Foundation of China (No. 81300022) and the Natural Science Foundation of Shanghai Science and Technology Commission (No. 13ZR1457700).

\section{Author Contributions}

J.P. conceived and designed research; J.P., X.-M.L., G.-R.Z., W.G., X.C., and Y.C. performed the experiments; J.P., X.-M.L., and X.-J.G. analyzed the data; J.P. and X.-M.L. interpreted the results of the experiments; X.-M.L. prepared the figures; J.P. and X.-M.L. drafted and revised the manuscript; J.P., X.-M.L., and X.-J.G. approved the final version of the manuscript.

\section{References}

1 Bostantzoglou C, Delimpoura V, Samitas K, Zervas E, Kanniess F, Gaga M. Clinical asthma phenotypes in the real world: opportunities and challenges. Breathe (Sheff). 2015 Sep; 11(3):186-93.

2 Wei Y, Dong M, Zhang H, Lv Y, Liu J, Wei K, et al. Acupuncture attenuated inflammation and inhibited Th17 and Treg activity in experimental asthma. Evid Based Complement Alternat Med. 2015;2015:340126.

3 Yun X, Shang Y, Li M. Effect of Lactobacillus salivarius on Th1/Th2 cytokines and the number of spleen $\mathrm{CD} 4^{+} \mathrm{CD} 25^{+}$Foxp $3^{+}$Treg in asthma Balb/c mouse. Int J Clin Exp Pathol. $2015 \mathrm{Jul} ; 8(7): 7661-74$.

4 Huang X, Tang L, Wang F, Song G. Astragaloside IV attenuates allergic inflammation by regulation Th1/Th2 cytokine and enhancement CD4(+)CD25(+)Foxp3 T cells in ovalbumin-induced asthma. Immunobiology. 2014 Jul;219(7):565-71.

5 Ji NF, Xie YC, Zhang MS, Zhao X, Cheng H, Wang H, et al. Ligustrazine corrects Th1/Th2 and Treg/Th17 imbalance in a mouse asthma model. Int Immunopharmacol. $2014 \mathrm{Jul}$ 21(1):76-81.

6 Holgate ST. Innate and adaptive immune responses in asthma. Nat Med. 2012 May;18(5): 673-83.

7 Erle DJ, Sheppard D. The cell biology of asthma. J Cell Biol. 2014 Jun;205(5):621-31.

8 Roussel L, Houle F, Chan C, Yao Y, Bérubé J, Olivenstein R, et al. IL-17 promotes p38 MAPK-dependent endothelial activation enhancing neutrophil recruitment to sites of inflammation. J Immunol. 2010 Apr;184(8): 4531-7.
9 Halwani R, Al-Muhsen S, Hamid Q. T helper 17 cells in airway diseases: from laboratory bench to bedside. Chest. 2013 Feb; 143(2): 494-501.

10 Wong CK, Ho CY, Ko FW, Chan CH, Ho AS, Hui DS, et al. Proinflammatory cytokines (IL17, IL-6, IL-18 and IL-12) and Th cytokines (IFN-gamma, IL-4, IL-10 and IL-13) in patients with allergic asthma. Clin Exp Immunol. 2001 Aug;125(2):177-83.

11 Laan M, Palmberg L, Larsson K, Lindén A. Free, soluble interleukin-17 protein during severe inflammation in human airways. Eur Respir J. 2002 Mar;19(3):534-7.

12 Bullens DM, Truyen E, Coteur L, Dilissen E, Hellings PW, Dupont LJ, et al. IL-17 mRNA in sputum of asthmatic patients: linking $\mathrm{T}$ cell driven inflammation and granulocytic influx? Respir Res. 2006 Nov;7(1):135.

13 Peng J, Yang XO, Chang SH, Yang J, Dong C. IL-23 signaling enhances Th2 polarization and regulates allergic airway inflammation. Cell Res. 2010 Jan;20(1):62-71.

14 Li XM, Peng J, Gu W, Guo XJ. TCDD-Induced Activation of Aryl Hydrocarbon Receptor Inhibits Th17 Polarization and Regulates Non-Eosinophilic Airway Inflammation in Asthma. PLoS One. 2016 Mar; 11(3):e0150551.

15 Faustman DL, Davis M. TNF receptor 2 and disease: autoimmunity and regenerative medicine. Front Immunol. 2013 Dec;4:478.

16 Chen X, Oppenheim JJ. The phenotypic and functional consequences of tumour necrosis factor receptor type 2 expression on CD4(+) FoxP3(+) regulatory $\mathrm{T}$ cells. Immunology. 2011 Aug;133(4):426-33.
17 Chen X, Oppenheim JJ. TNF-alpha: an activator of $\mathrm{CD} 4+\mathrm{FoxP} 3+\mathrm{TNFR} 2+$ regulatory $\mathrm{T}$ cells. Curr Dir Autoimmun. 2010;11:119-34.

18 Candel S, de Oliveira S, López-Muñoz A, García-Moreno D, Espín-Palazón R, Tyrkalska $\mathrm{SD}$, et al. Tnfa signaling through tnfr2 protects skin against oxidative stress-induced inflammation. PLoS Biol. 2014 May; 12(5):e1001855.

19 Chen Z, Palmer TD. Differential roles of TNFR1 and TNFR2 signaling in adult hippocampal neurogenesis. Brain Behav Immun. 2013 May;30:45-53.

20 Li XM, Chen X, Gu W, Guo YJ, Cheng Y, Peng $J$, et al. Impaired TNF/TNFR2 signaling enhances Th 2 and Th17 polarization and aggravates allergic airway inflammation. Am J Physiol Lung Cell Mol Physiol. 2017 Sep; 313(3):L592-601.

21 Gregori S, Tomasoni D, Pacciani V, Scirpoli M, Battaglia M, Magnani CF, et al. Differentiation of type $1 \mathrm{~T}$ regulatory cells $(\mathrm{Tr} 1)$ by tolerogenic DC-10 requires the IL-10-dependent ILT4/HLA-G pathway. Blood. 2010 Aug; 116(6):935-44.

22 Aggarwal BB. Signalling pathways of the TNF superfamily: a double-edged sword. Nat Rev Immunol. 2003 Sep;3(9):745-56.

23 Gordon C, Ranges GE, Greenspan JS, Wofsy $D$. Chronic therapy with recombinant tumor necrosis factor-alpha in autoimmune NZB/ NZW F1 mice. Clin Immunol Immunopathol. 1989 Sep;52(3):421-34.

24 Jacob CO, Aiso S, Michie SA, McDevitt HO, Acha-Orbea H. Prevention of diabetes in nonobese diabetic mice by tumor necrosis factor (TNF): similarities between TNF-alpha and interleukin 1. Proc Natl Acad Sci USA. $1990 \mathrm{Feb} ; 87(3): 968-72$. 
25 Ko JM, Gottlieb AB, Kerbleski JF. Induction and exacerbation of psoriasis with TNFblockade therapy: a review and analysis of 127 cases. J Dermatolog Treat. 2009;20(2):100-8.

26 Mohan N, Edwards ET, Cupps TR, Oliverio PJ, Sandberg G, Crayton H, et al. Demyelination occurring during anti-tumor necrosis factor alpha therapy for inflammatory arthritides. Arthritis Rheum. 2001 Dec;44(12): 2862-9.
27 Wu AJ, Hua H, Munson SH, McDevitt HO. Tumor necrosis factor- $\alpha$ regulation of CD4+CD25+ T cell levels in NOD mice. Proc Natl Acad Sci USA. 2002 Sep;99(19):1228792.

28 Chen X, Bäumel M, Männel DN, Howard OM, Oppenheim JJ. Interaction of TNF with TNF receptor type 2 promotes expansion and function of mouse CD4+CD25+ T regulatory cells. J Immunol. 2007 Jul;179(1):154-61.
29 Maier O, Fischer R, Agresti C, Pfizenmaier K. TNF receptor 2 protects oligodendrocyte progenitor cells against oxidative stress. Biochem Biophys Res Commun. 2013 Oct;440(2):33641.

30 Madsen PM, Motti D, Karmally S, Szymkowski DE, Lambertsen KL, Bethea JR, et al. Oligodendroglial TNFR2 Mediates Membrane TNF-Dependent Repair in Experimental Autoimmune Encephalomyelitis by Promoting Oligodendrocyte Differentiation and Remyelination. J Neurosci. 2016 May;36(18):512843. 